\title{
Searches for New Physics with Lepton Flavours and Multi-lepton Final States in ATLAS
}

\author{
Romain Madar ${ }^{1, a}$ on behalf of the ATLAS collaboration \\ ${ }^{1}$ Université Clermont Auvergne, Université Blaise Pascal, CNRS/IN2P3, Laboratoire de Physique Corpus- \\ culaire, BP 10448, F-63000 CLERMONT-FERRAND, France
}

\begin{abstract}
Lepton flavours and multi-lepton signatures are powerful probes to search for new physics. Also, the lepton sector of the Standard Model has to be extended to describe the observed neutrino oscillations. ATLAS experiment searches for lepton flavour violation, new gauge boson allowing to unify quark and lepton fields, heavy charged and neutral leptons as well as for new phenomena in multiphoton final states are summarized in this document. The most recent results will be discussed and compared with existing results.
\end{abstract}

\section{Introduction}

The leptonic sector of the Standard Model (SM) is a promising area to search for new phenomena beyond the SM (BSM). In a bottom-up approach, the neutrino oscillations is probably one of the best established and mesured BSM observation. This phenomena suggests that neutrinos are massive but the mechanism which induces neutrino masses remains an open question. Another consequence of the neutrino oscillation is the violation of lepton flavour in the neutral lepton sector. Searching for lepton flavor violation (LFV) in the charged sector becomes then a natural approach to better identify a new physics beyond what is currently known. Also, the LFV might be also connected to one of the most fundamental open question: the matter-antimatter asymmetry observed in our Universe [1]. In a top-down approach, the leptonic sector is involved in Grand Unified Theories (GUT) which predict new particles. One of the key ideas is to consider that quarks and leptons are two components of a unique, larger, mathematical object. This can be realised using larger groups of symmetry which lead to new gauge bosons coupled to leptons but also to quarks. In all the SM extensions addressing these issues, new states involving leptons are predicted and could be produced at the LHC.

\section{Lepton Flavour Violation in $Z$ Boson Decay}

The LFV is observed in the neutral lepton sector via neutrino oscillations phenomenon. It can be searched at the LHC in the charged lepton sector using the $Z \rightarrow e \mu$ decay signature. In the SM, the level of LFV in the charged lepton sector is highly suppressed. Typically, the SM predicts $\mathcal{B R}(\mu \rightarrow$ e $\gamma) \sim 10^{-54}$ [2], which is well below any experimental sensitivity. An observation

\footnotetext{
ae-mail: romain.madar@cern.ch
} 
of LVF in the charged sector would lead to a clear signature for BSM physics. Experimental upper limits on $\mathcal{B R}(Z \rightarrow e \mu)$ were obtained at $\operatorname{LEP}\left(<1.710^{-6}\right)$ but also indirectly using $\mu \rightarrow 3 e$ constraints reintrepreted in term of $Z^{*} \rightarrow e \mu$ decay $\left(<10^{-12}\right)$. The ATLAS result [3] is based on an analysis of collisions where an isolated electron and muon are reconstructed, with an invariant mass compatible with the Z-boson mass. The signal would then appear as a mass-localised excess of events. The SM backgrounds are mainly reducible. The $W W \rightarrow e v_{e} \mu v_{\mu}$ production will produce a large missing transverse momentum $\left(E_{\mathrm{T}}^{\text {miss }}\right)$ due to escaping neutrinos coming from $W$-boson decay, as shown in Figure 1. The top quark pair production can be suppressed disgarding collision with a large number of jets. The only irreducible background is the $Z \rightarrow \tau \tau$ process where each tau lepton decays into a muon and a electron respectively. No excess of events is found on top of the expected SM background and an upper limit at $95 \%$ confidence level (CL) on the number of signal events is obtained based on an unbinned fit of data to the $(e, \mu)$ invariant mass distribution as shown in Figure 1. This upper limit is then converted into a limit on the branching ratio $\mathcal{B R}(Z \rightarrow e \mu)$. The obtained constraint reaches $7.510^{-7}$ and is twice better than LEP direct limit.
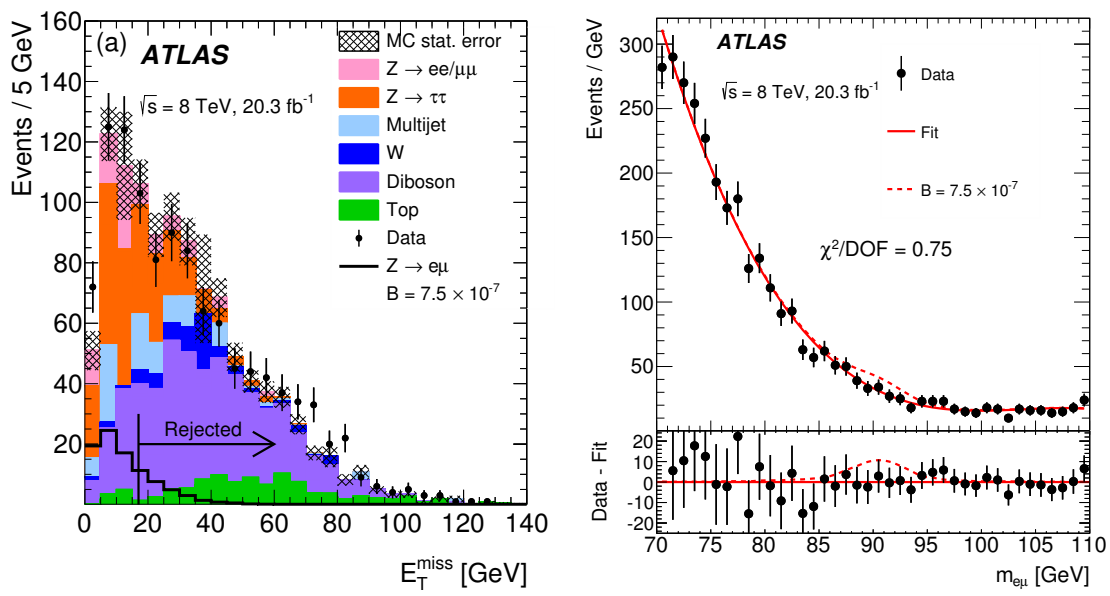

Figure 1. Missing transverse energy distribution (left) and invariant mass distribution of the $(e, \mu)$ system (right). Taken from Ref. [3].

Being a BSM phenomenon, it is natural to expect that LFV migh also occur in new neutral resonance sectors. ATLAS obtained constraints on this possible LFV signature [4] based on two benchmarks scenarios: a supersymmetric extension of the SM where the new resonance is a scalar $\left(\tilde{v}_{\tau}\right)$ and an additional gauge sector with a new vectorial field $\left(Z^{\prime}\right)$. The strategy is the same as in [3] except that all the three possibles final states are analysed namely $e \mu, e \tau, \mu \tau$ where tau lepton decay in hadrons. Final states with hadronically decaying tau leptons lead to two specific experimental challenges. First, the background due to parton-initiated jets faking hadronic tau leptons can be large and is particularly difficult to accurately model using simulated collisions. The prediction of this background has to be extracted from real data in relevant signal-free control regions. Second, the reconstuction of the mass of the resonance cannot be fully performed because of the neutrinos coming from tau leptons decay. To cope with this issue, neutrino kinematics can be deduced from the missing transverse momentum and assuming they are emitted in the same direction as visible tau decay products - this assumption being valid for high masse resonances. 

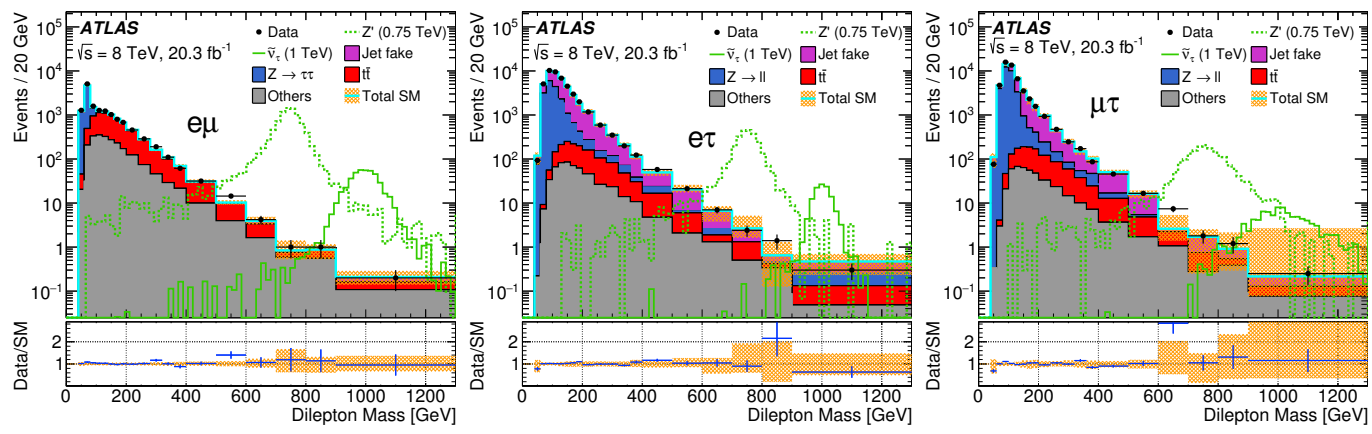

Figure 2. Dilepton invariant mass distribution for the $e \mu$ (left), $e \tau$ (middle) and $\mu \tau$ (right) final state. The expected background, observed data and expected signals (for the two considered benchmarks) are shown. Taken from Ref. [4].

Figure 2 shows the invariant mass of the $\left(\ell, \ell^{\prime}\right)$ system for the three considered final states. The large contamination from parton-initiated jets is clearly visible for the $e \tau$ and $\mu \tau$ channels. Since no excess is observed in data, upper limits on the signal cross-section are extracted for the three different channels and the two benchmark signals. Thes excluded cross-section can be interpreted in excluded mass for the new resonances using the prediction of the two particular models. The typical excluded mass scale goes from 1.7 to $2.5 \mathrm{TeV}$. The CMS experiment has similar results [5] although a proper comparison is difficult due to significant differences in the theoretical benchmarks.

\section{Leptoquarks Searches}

The leptoquarks $(L Q)$ are colored gauge bosons predicted by many GUTs attempting to unify lepton and quark fields in a same group representation. The parameter space of these theories is quite large but can be highly reduced by exisiting constraints on flavour changing neutral currents (FCNC) and on the proton lifetime. After taking these constraints into account, the phenomenology of these SM extensions includes three generations of leptoquarks corresponding to the three generation of fermions $\left(L Q_{i}, i=\{1,2,3\}\right)$ - all other structure being strongly constrained by FCNC. These bosons can decay into either a charged lepton and quark or into a neutrino and a quark - all other couplings being strongly constrained by the proton lifetime. Experimentally, the relevant decay mode is the visible one $L Q_{i} \rightarrow \ell_{i} q_{i}$ and its branching ratio is written $\beta$.

At the LHC, leptoquarks can be pair or single produced. The search performed by ATLAS experiment [6] focuses on pair-produced leptoquarks of first and second generation ${ }^{1}$ in $e e j j$ and $\mu \mu j j$ final states respectively, the main SM background being $Z+$ jets production. The search strategy is based on the specific topology of leptoquarks pair production. First, the high mass of these new gauge bosons lead particles with high transverse momentum $\left(p_{\mathrm{T}}\right)$ in the final state. This property helps to discriminate the signal from SM backgrounds with the distribution of $S_{\mathrm{T}}$, defined as the scalar $p_{\mathrm{T}}$ sum of the two leading leptons and two leading jets, as shown in Figure 3. Second, the mass of the produced leptoquarks can be partially reconstructed using $(\ell, j)$ pairing combinatorics - each $(\ell, j)$ system coming from $L Q$ decay having the same invariant mass.

\footnotetext{
${ }^{1}$ The third generation can be probed using bottom quark and top quark final states but the strategy is slightly different and this analysis is not detailed in this document.
} 

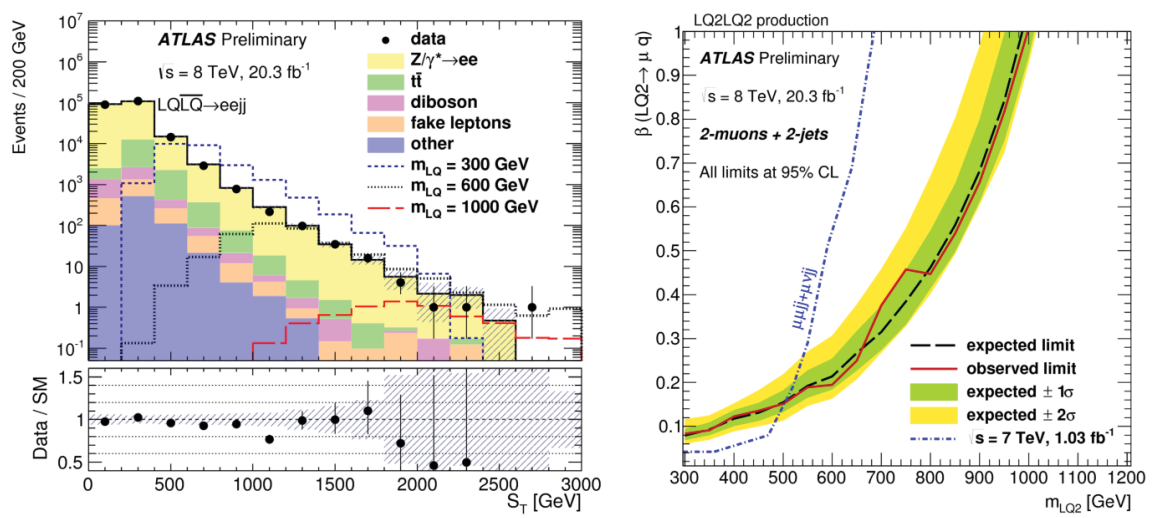

Figure 3. Distribution of scalar $p_{\mathrm{T}}$ sum for the $e e j j$ final state (left) and excluded signal at $95 \%$ confidence level in the $(\beta, m)$ parameter space for the $\mu \mu j j$ final state (right). Taken from Ref. [6].

Since no excess of data is observed, $L Q$ must be heavy or weakly coupled to SM particles if they are realised in Nature. An upper limit at 95\% C.L. on the cross-section of $L Q$ pair production is obtained based on cut-and-count analysis in several signal regions tuned separately for each mass hypothesis. This limit can be interpreted in term of constraints in the model parameter space, namely the leptoquark mass $\left(m_{L Q}\right)$ and the branching ratio of $L Q$ decay into $\ell j$. Figure 3 shows the obtained constraint in the $\left(m_{L Q}, \beta\right)$ plane for the second generation leptoquarks. Overall, the typical excluded leptoquark mass scale is $1 \mathrm{TeV}$. CMS experiment has similar results [7] although a search for single $L Q$ production only were performed in the electron channel.

\section{Heavy Lepton Searches}

Neutrino masses, offering the simplest explanation of flavour oscillations, are not described in the SM and can also be solved by BSM dynamics which predict new heavy leptonic states. ATLAS experiment performed searches for three types of heavy leptons exploiting several final states $[8,10,11]$.
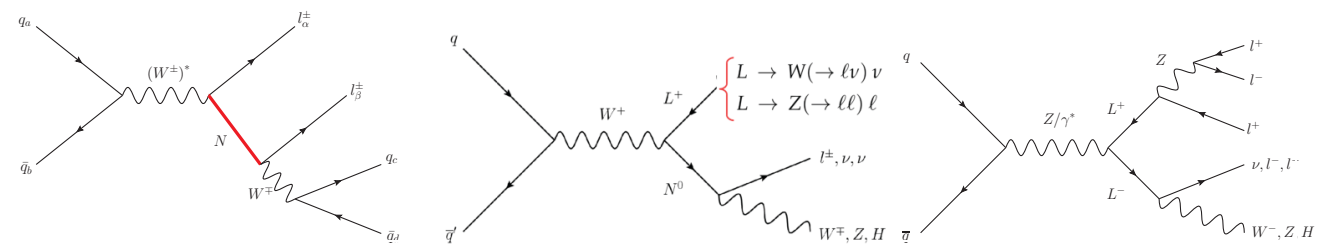

Figure 4. Distribution of scalar $p_{\mathrm{T}}$ sum for the $e e j j$ final state (left) and excluded signal at $95 \%$ confidence level in the $(\beta, m)$ parameter space for the $\mu \mu j j$ final state (right).

The first type is a generic heavy Majorana neutrino $N$ appearing in two different classes of model: type-I seesaw mechanism allowing generating neutrino masses and left-right symmetric models (where left-handed and right-handed fermions interact in the same way) having additionnal gauge 
bosons $\left(W_{R}^{\prime}, Z^{\prime}\right)$. The second type of heavy lepton consists in a weak-triple lepton $\left(L^{+}, N^{0}, L^{-}\right)$involved in type-III seesaw mechanism, and the last type is a heavy lepton having only vectorial couplings (vector-like lepton) which are predicted by many BSM theories like composite Higgs boson or wraped extra-dimensions. Figure 4 shows examples of feynman diagrams for the different signals process which could occurs at the LHC.

ATLAS experiment focused on heavy Majorana neutrino search using two leptons having the same electric charge [8], as showns in first diagram of Figure 4. The particular signal topology allows reducing the SM background, dominated by charge mis-reconstruction and mis-identification of leptons. In the type-I seesaw signal, the hardonic decay of the $W$ boson makes the di-jet mass peaking at $m_{W}$. In the case of the left-right symmetric model, the heavy $Z^{\prime}$ is produced in a s-channel and follows the decay chain

$$
p p \rightarrow Z^{\prime} \rightarrow N N \text { and } N \rightarrow W_{R}^{ \pm \prime}(\rightarrow j j) \ell^{ \pm}
$$

The $Z^{\prime}$ mass can be then reconstructed from the invariant mass of all objects in the final state. Since no excess is observed in data, upper limits on cross-section can be obtained and as well as a reinterpretation in term of model parameters. Figure 5 shows the region of the left-right symmetric model parameters which are excluded by ATLAS experiment. In summary, Majorana neutrino is excluded up to $300 \mathrm{GeV}$ for a given coupling to lepton $\left|V_{\ell N}\right|^{2}=10^{-1}$ (the excluded region in the mass-coupling plane is also derived). CMS experiment obtained better constraint but analyzed the muon channel only [9].
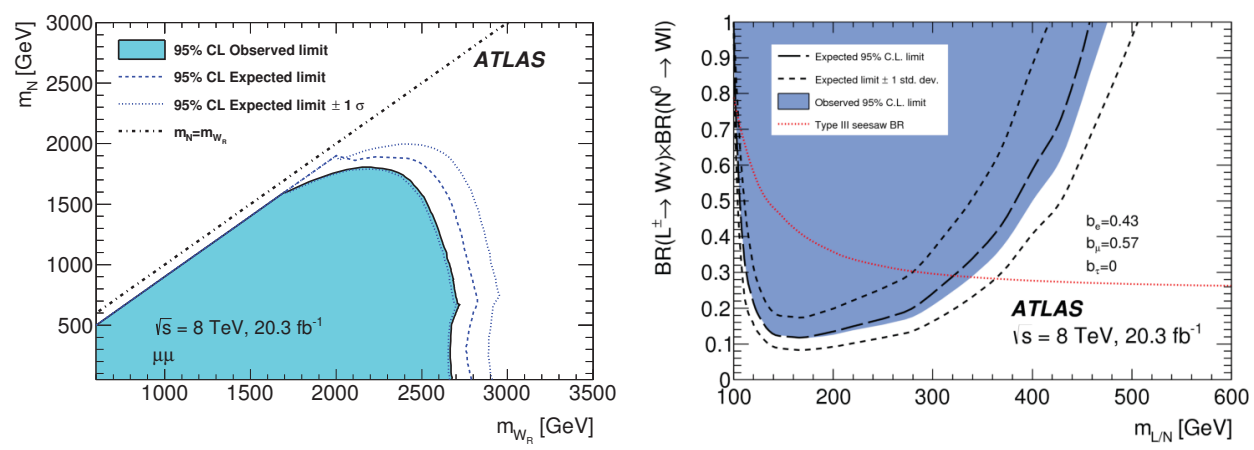

Figure 5. Excluded region at 95\% C.L. for the left-right symmetric model (left) and type-III seesaw mechanism (right). Taken from Ref. [8].

The models based on a triplet of heavy leptons with $L \rightarrow W v$ have two type of experimental signatures, as shown in the second diagram of Figure 4. One contains two same-sign leptons with two jets and the other has two oppsite-sign leptons with tow jets, coming from the $W$ boson decay in each case. Event selection is optimized for each of the six categories to increase the signal over background ratio. Since no excess is observed in data, an upper limit were obtained and reinterpreted in constraints of term of model parameters. Figure 5 shows the excluded regions the $\left(m_{N / L}, \mathcal{B R}_{L \rightarrow W v} \times \mathcal{B R}_{N \rightarrow W \ell}\right)$ plane, where $m_{N / L}$ is the mass of the triplet. The typical excluded mass scale is reaches $350 \mathrm{GeV}$ for this type of heavy leptons.

It is possible to extend the search to the case where $L \rightarrow Z \ell$ by considering several multi-lepton final states [11]. Models based on vector-like lepton can also lead to the same kind of experimental 
signature. In this case, there are 6 signal regions can be analyzed to probe the presence of signal. Since no excess is observed, upper limits are derived and typical excluded mass scales reach $400 \mathrm{GeV}$ for type-III seesaw leptons and $160 \mathrm{GeV}$ for vector-like leptons.

\section{Multi-photons Final States Searches}

The search for new physics in multi-photon final states is also connected to rare $Z$ decays and more generally to new dynamics in the lepton sector. The lepton flavor violation in $\mathrm{Z}$ decay was discussed in section 2 , but this is not the only way $Z$ boson properties can be affected by new physics. Indeed, a $Z$ decay into three photons would be clear signature of new physics as it is highly suppressed in the $\mathrm{SM}\left(\mathcal{B R}(Z \rightarrow 3 \gamma) \sim 10^{-10}\right)$. In addition, many BSM models predict multi-photon final state such as nMSSM predicting a pseudo-scalar boson $a$ coupled to Higgs bosons and photons or additional gauge sector extensions predicting $Z^{\prime}$ coupled to $a$ and photon fields. Figure 6 shows the feynman diagrams corresponding to the signal processes of these SM extensions at the LHC, which are searched for by ATLAS experiment [12].
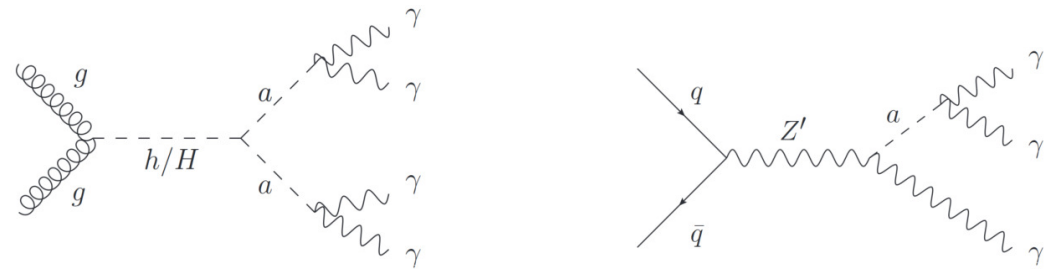

Figure 6. Feynman diagrams corresponding to the signal in the nMSSM (left) and in case of new gauge sector (right).

In this kind of final state, the main background comes from parton-initiated jets that are wrongly reconstructed as photons. Being challenging to accurately model using simulation, the prediction of this SM background is obtained using real data in signal-free control regions. The search strategies are different for $Z \rightarrow 3 \gamma$ search and for new resonances search. The first one is based on count of events with three well isolated photons having an invariant mass compatible with the $Z$-boson mass. For resonnances searches, a localised excess of event is searched for in each photon pair invariant mass spectrum, as shown in Figure 7. There is no evidence for new physics in both cases and upper limits are then derived. The constraint on $\mathcal{B R}(Z \rightarrow 3 \gamma)$ reaches the level of $2.210^{-6}$, which is five times better than LEP limit. Concerning the $a$ and $Z^{\prime}$ resonance searches, the excluded production cross-section is of the order of the $1 \mathrm{fb}$ for $a$-boson mass between 10 and $240 \mathrm{GeV}$ and a $Z^{\prime}$-boson mass between 100 and $500 \mathrm{GeV}$, as shown in Figure 7. These are the first constraints on this type of models. 

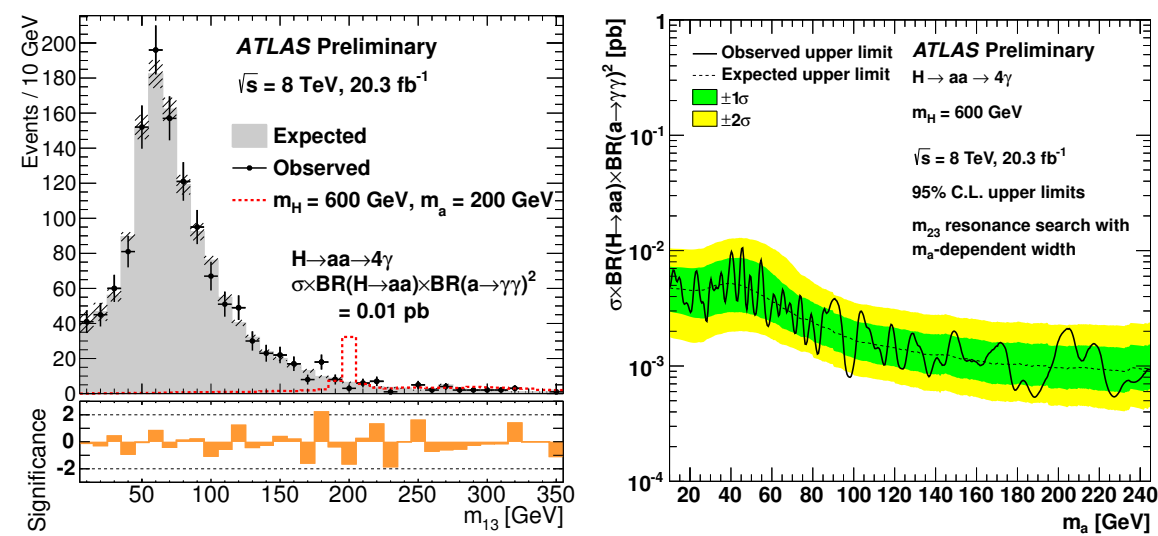

Figure 7. Distribution of invariant mass of the leading and third-leading photon system (left) and upper limit on cross-section production for nMSSM signal (right). Taken from Ref. [12].

\section{Summary}

The lepton sector of the SM is very promising to search for new physics given the observed neutrino oscillations, and could possibly address the matter-antimatter asymmetry problem. Search for lepton flavor violation in the SM were pushed twice below existing LEP limits and LFV beyond the SM sector is now excluded to typical mass scale of $2.5 \mathrm{TeV}$. The interesting approach of unifying quarks and leptons predicting new gauge bosons is also now excluded up to a typical mass scale of $1 \mathrm{TeV}$. Heavy leptons that could explain neutrino oscillations are now constrained to be typically heaviest than $300 \mathrm{GeV}$. Finally, multiphoton final states allow constraining rare Z-boson decay in three photon to the level of $10^{-6}$ which is five times better than LEP limit. It also provides the first constraints on particular BSM signals leading to multiphoton final states.

\section{References}

[1] M. Fukugita and T. Yanagida, Phys. Lett. B. 174, 45 (1986)

[2] S. M. Bilenky, S.T. Petcov, B. Pontecorvo, Phys. Lett. B 67309 (1977)

[3] ATLAS Collaboration, Phys. Rev. D. 90, 072010 (2014)

[4] ATLAS Collaboration, Phys. Rev. Lett. 115, 031801 (2015)

[5] CMS Collaboration, PAS EXO-13-002 (2015)

[6] ATLAS Collaboration, arXiv:1508.04735 [hep-ex] (2015), submitted to EPJC

[7] CMS Collaboration, arXiv:1509.03750 [hep-ex] (2015), submitted to PRD

[8] ATLAS Collaboration, JHEP 07162 (2015)

[9] CMS Collaboration, Phys. Lett. B 748, 144-166 (2015)

[10] ATLAS Collaboration, JHEP 09108 (2015)

[11] ATLAS Collaboration, Phys. Rev. D. 92032001 (2015)

[12] ATLAS Collaboration, arXiv:1509.05051 [hep-ex] (2015), submitted to EPJC 